\title{
Measurement of Bacterial Flagellar Thrust by Negative Dielectrophoresis
}

\author{
Michael P. Hughes and Hywel Morgan* \\ Bioelectronics Research Centre \\ University of Glasgow \\ Glasgow G12 8QQ \\ Scotland, UK
}

* Author to whom correspondence should be addressed. 


\begin{abstract}
The force produced by the flagella of the bacterium Salmonella typhimurium has been measured using negative dielectrophoretic methods. The bacteria are held in a force funnel, produced using a non-uniform electric field. When the motor force is balanced against an opposing negative dielectrophoretic force the bacteria become motionless. Numerical simulations have been used to estimate the electric field gradient in the electrodes. Together with experimental observations of bacterial motion the data gives a value of the force produced by the bacterial motor to be $0.37 \mathrm{pN}$.
\end{abstract}




\section{Introduction}

Many types of bacteria are able to actively move around their aqueous environment through the use of spiral organs or flagella. A bacterial flagellum is a rigid helical protein filament approximately $20 \mathrm{~nm}$ in diameter, extending some $10 \mu \mathrm{m}$ from the bacterial wall. The flagellum rotates in a "corkscrew" motion at between $20-100 \mathrm{~Hz}$, acting as an Archimedes screw driving the bacterium forward. Typical velocities can reach $50 \mu \mathrm{m} / \mathrm{sec}$, although some species can move at over $600 \mu \mathrm{m} / \mathrm{sec}$ (for review, see ref [1]). The bacterial flagellum is driven at its base by a molecular rotary motor driven by a proton gradient and acting in a manner similar to a stepper motor $[1,2]$ Interest in the function of such molecular motors is growing, not least in part due to the possibility of replicating such motors for use in molecular nanotechnology [3].

Recent studies have used different physical techniques to investigate the mechanical characteristics of this bacterial motor. Block et al [4] used laser tweezers to determine the flagellar compliance, and Washizu et al [5] used dielectrophoresis to determine the dipole induced along the length of the flagellum. More recently the electrorotation of "tethered" bacteria was measured to determine the torque produced by the bacterial motor [6-8]. DEP was also used to apply a "load" to a moving bacterium and thus determine the force-load characteristics [6]. In this paper we show that it is possible to make quick and accurate measurements of the force generated by the flagellum using DEP methods.

Dielectrophoresis (DEP) is the term given to the force experienced by a polarised particle in a non-uniform electric field [9]. This force can act either toward or away from electric field maximum depending on the sign of the polarisability of the particle 
with respect to the suspending medium. Positive DEP occurs when the force is towards high electric field regions, and vice versa for negative DEP. Since the polarisability is a factor which depends on both the frequency and the particle properties, DEP has been used as a means of characterising different types of bacteria [10], and also separate different strains according to their different biophysical makeup [11].

Using an electrode of suitable geometry, a spatially-dependent DEP force funnel has been generated. The bacteria move randomly within this force funnel until the forward force generated by the motor is exactly equal and opposite to the DEP force and the bacteria become motionless. Thus the bacterial motor force can be obtained. In earlier work, positive DEP was used to estimate the force produced by the bacterial motor [6]. However, that method was confounded by directional instability problems and is effective only when bacteria swim directly along the field lines. By using negative DEP traps, this problem is eliminated. Furthermore, using negative DEP measurements can be performed in high conductivity media (such as growth media) because in this case the organisms experience negative DEP across all frequencies.

\section{Theory}

A dielectrophoretic force occurs due to the interaction of an induced dipole and an electric field gradient. The time averaged force FDEP on a homogeneous dielectric sphere, [12] is:

$$
\mathbf{F}_{\mathbf{D E P}}=\operatorname{Re}[K(\omega)] 2 \pi_{r}^{3} \varepsilon_{m} \nabla \mathbf{E}_{r m s}^{2}
$$


where $r$ is the particle radius, $\varepsilon_{m}$ is the absolute permittivity of the suspending medium, $\nabla$ the del vector operator, $\mathbf{E}_{r m s}$ is the local RMS electric field and $\operatorname{Re}[K(\omega)]$ is the real part of the Clausius-Mossotti factor, given by:

$$
K(\omega)=\frac{\varepsilon_{p}^{*}-\varepsilon_{m}^{*}}{\varepsilon_{p}^{*}+2 \varepsilon_{m}^{*}}
$$

where $\varepsilon_{m}{ }^{*}$ and $\varepsilon_{p}{ }^{*}$ are the complex permittivities of the medium and particle respectively. A general permittivity is defined as $\varepsilon^{*}=\varepsilon-j \frac{\sigma}{\omega}$ with permittivity $\varepsilon$ and conductivity $\sigma$. The direction of the force (positive or negative DEP) is governed by the value of $\operatorname{Re}[K(\omega)]$, which can take positive or negative values respectively depending on the applied frequency or the particle and suspending medium conductivity and permittivity.

A bacterium moving through a suspending medium is impeded by viscous drag. In the absence of a trailing flagellum, Stokes' equation indicates the bacterium will reach a terminal velocity $\mathbf{v}_{\mathbf{T}}$ given by:

$$
\mathbf{v}_{\mathbf{T}}=\frac{\mathbf{F}_{\mathbf{D E P}}}{f}
$$

where $f$ is the friction factor, which depends on the medium viscosity and the geometry of the particle. If a load is applied such that the velocity is zero, the factor $f$ is zero and a measurement of the force can be taken directly. Such a situation occurs when a bacterium is suspended in a dielectrophoretic force field such that its forward motion is exactly countered by the retarding DEP force. 
In this work we have used a "polynomial" electrode design [13] to create a welldefined DEP force gradient as a means of measuring the force produced by the bacteria. A photograph of the electrode is shown in figure 1. The negative-DEP is greatest at the electrode edges so that the bacteria are generally pushed into the middle of the electrode. Any bacteria in the force funnel swim up the DEP force gradient to a balance point at which the driving force provided by the motor is equal and opposite to the retarding dielectrophoretic force. At this point the bacteria become motionless. Numerical simulations of the electric field gradient were used to estimate the electric field gradient as a function of radius from the electrode centre so that the force produced by the flagella could be estimated. This method is in principle similar to that employed in [14] to obtain the conditions for stable trapping of nanometre-scale bioparticles.

\section{Material and Methods}

\subsection{Bacteria Preparation}

Salmonella typhimurium NCTC 0073 were supplied by Mr Ian Watt at the IBLS teaching unit, University of Glasgow and cultures were incubated for 24 hours at $37^{\circ} \mathrm{C}$ in $10 \mathrm{ml}$ brain/heart infusion (Oxoid CM225) prior to experimentation.

\subsection{Experimental}

Microelectrodes were fabricated using standard photolithographic techniques. Glass microscope slides were used as substrates and the electrodes consisted of a $100 \mathrm{~nm}$ thick $\mathrm{Au}$ layer deposited over a 10nm Ti seed layer. The electrodes were manufactured with a $25 \mu \mathrm{m}$ gap in the centre, and a minimum distance of $5 \mu \mathrm{m}$ 
between adjacent electrode edges. The electrodes were powered using a computercontrolled Direct Digital Synthesis (DDS) function generator providing sinusoidal signals of up to $12 \mathrm{~V}_{\mathrm{pk}}$ across the frequency range $1 \mathrm{kHz}-20 \mathrm{MHz}$. Experiments were observed using a Nikon Microphot microscope, connected to a Panasonic CCD camera and images were recorded using S-VHS video.

\section{Results and Discussion}

\subsection{Experimental Results}

The bacteria were taken directly from the $37^{\circ} \mathrm{C}$ incubator immediately prior to experimentation and a $30 \mu \mathrm{l}$ volume pipetted onto the electrode array, which was at a temperature of $25^{\circ} \mathrm{C}$. At this temperature, the motility of the bacteria was reduced, but they exhibited quite strong and continuous swimming movements for over 30 minutes at this temperature. Bacteria swimming speeds were measured at approximately $25-30 \mu \mathrm{ms}^{-1}$.

AC potentials were applied at a frequency of $1 \mathrm{MHz}$, so that the bacteria experience negative DEP. At this frequency, and for an applied potential of greater than $2 \mathrm{~V}_{\mathrm{pk}}$, the bacteria were forced into the centre of the trap in a similar manner to that described for other organisms undergoing negative DEP in these electrodes, e.g. [13]. Bacteria confined within this region attempted to swim radially out from the centre. However, at a given radius the motile force of the bacterium became equal and opposite to the DEP force so that the bacteria appeared to swim up to a fixed but invisible boundary. The radius of this boundary varied with the applied voltage. 
Figures 2 (a) to (f) are images of bacteria on the electrode array and show typical patterns of behaviour for a range of applied potentials between $1 \mathrm{~V}_{\mathrm{pk}}$ and $10 \mathrm{~V}_{\mathrm{pk}}$. In this figure the white lines represent the average outer boundary beyond which bacteria did not swim. As can be seen, the size varies as a function of the applied potential. No collection was observed for $1 \mathrm{~V}_{\mathrm{pk}}$ applied signals, since presumably the magnitude of the dielectrophoretic force was insufficient to hold the bacteria within the confines of the electrodes. At an applied potential of $2 \mathrm{~V}_{\mathrm{pk}}$, the DEP force at the centre of the electrode tips was sufficient for some bacteria to become trapped in the centre. At this voltage, bacteria were occasionally observed to swim through the trap and escape along the upper electrode surfaces. This only happened when a bacterium was moving straight towards the electrode edge. At higher voltages this did not happen; the bacteria in the electrode vicinity were forced into an approximately circular pattern. At a voltage of $10 \mathrm{~V}_{\mathrm{pk}}$ the bacteria were observed to levitate at the centre of the chamber, figure 2 (f).

\subsection{Electric Field Modelling}

Computer simulations of the electric field in the polynomial electrode were obtained using the 2D finite element field modelling software, Maxwell (Ansoft, Inc). The post-processor was used to estimate both the electric field and the field gradient, and thus the DEP force experienced by a bacterium across the electrode chamber. The simulation used 1137 elements to cover the electrode chamber and was solved to $0.04 \%$ error in energy.

A plot of the magnitude of the electric field together with $\nabla \mathbf{E}_{r m s}^{2}$ (proportional to the DEP force) is shown in figure 3 (a) and (b) respectively. These plots were calculated 
for an applied potential of $1 \mathrm{~V}_{\mathrm{pk}}$. The DEP force contours (indicated by a band of constant brightness) are similar to the patterns of the bacteria trapped within the electrode chamber. The DEP force (equation (1)) is proportional to $\mathbf{E}_{r m s}^{2}$, so that doubling the applied potential quadruples the DEP force experienced by the bacteria (at any given point in the electrode array). However, the profile of the force distribution remains the same. By comparing figures 2 and 3(b), it can be seen that the outline of the bacteria closely resembles the contours of constant $\nabla \mathbf{E}_{r m s}^{2}$.

The maximum force produced by the bacteria can be calculated from the value of $\nabla \mathbf{E}_{r m s}^{2}$ at the boundary of the bacterial collection pattern. The average radius of the collection pattern was measured as a function of applied potential and the data tabulated (table 1). Measurements were made along a radial line $a$ - $a$ as shown in figure 1. For each value of applied voltage, values of $\nabla \mathbf{E}_{r m s}^{2}$ were calculated at the measured radii, and this data is also shown in table 1. For applied potentials ranging from 2 to $5 \mathrm{~V}_{\mathrm{pk}}, \nabla \mathbf{E}_{r m s}^{2}=1.4 \times 10^{15} \mathrm{~V}^{2} \mathrm{~m}^{-3}$. However, at $10 \mathrm{~V}_{\mathrm{pk}}$, this increased to $5.6 \times 10^{15} \mathrm{~V}^{2} \mathrm{~m}^{-3}$. This increase could be due to the fact that the trapped bacteria are compressed together into a ball rather than lying flat on the electrode chamber surface. Also, as the DEP force increases the particles tend to levitate, and in doing so the DEP force they experience reduces compared with that predicted by the 2-D simulation.

Washizu et al (1993) estimated the value of the dielectrophoretic force (and thus $\nabla \mathbf{E}_{r m s}^{2}$ ) required to render a bacterium stationary using positive DEP forces. In their case, the value of $\nabla \mathbf{E}_{r m s}^{2}$ was obtained by approximating the electric field to the 
analytical solution for a coaxial system. Their calculations showed that for $S$. typhimurium the zero velocity point occurred for field gradients of $\nabla \mathbf{E}_{r m s}^{2}=1.25 \times 10^{15}$ $\mathrm{V}^{2} \mathrm{~m}^{-3}$. In the present work, using a negative dielectrophoretic force, then $\nabla \mathbf{E}_{r m s}^{2}=$ $1.4 \times 10^{15} \mathrm{~V}^{2} \mathrm{~m}^{-3}$ (for potentials in the range 2 to $5 \mathrm{~V}$ pk in close agreement with that measured by Washizu et al [6].

\subsection{Determination of Motor Force}

In the absence of detailed measurements of the dielectric properties of $S$. typhimurium, we have used the published dielectric data for Escherichia coli [15], a bacterium which is physically very similar to of $S$. typhimurium, to calculate the frequency dependence of $K(\omega)$. Two models have been considered; firstly, a multishell model suggested by Asami et al [16] in which a bacterium is modelled as a spherical particle with a cytoplasm, membrane and cell wall. The relative permittivity of the cytoplasm and cell wall were set equal to 60 , whilst the membrane was assigned a value of 10 . Conductivity values were set as follows: cytoplasm $=$ $0.19 \mathrm{~S} / \mathrm{m}$, cell wall $=0.9 \mathrm{~S} / \mathrm{m}$ and membrane $=5 \times 10^{-8} \mathrm{~S} / \mathrm{m}$. The growth medium used in this work had a conductivity of $\sigma_{m}=1.58 \mathrm{Sm}^{-1}$, so that at a frequency of $1 \mathrm{MHz}$ $K(\omega)=-0.488$.

The second model considered was that of a homogeneous dielectric sphere suggested by Berg and Turner [7], where the sphere has internal conductivity $40 \mathrm{mSm}-1$ and relative permittivity 600 . Under the same conditions as described above, this model predicts that at a frequency of $1 \mathrm{MHz}, K(\omega)=-0.482$, a similar value to that provided by the previous model. In order to test the correlation further, the multi-shell model was tested against the electrorotation spectra for E. Coli described in [7]; for $0.4 \mathrm{mSm}^{-1}$ 
solution conductivity, rotation peaks were predicted at $0.97 \mathrm{MHz}$ and $1.3 \mathrm{MHz}$ for the solid and multi-shell models respectively, both in keeping with the experimental results described in [7]. Substituting the values of $K(\omega)$ into equation (1) and putting $\nabla \mathbf{E}_{r m s}^{2}=1.4 \times 10^{15} \mathrm{~V}^{2} \mathrm{~m}^{-3}, r=0.5 \mu \mathrm{m}$ and $\varepsilon_{\mathrm{m}}=78 \varepsilon_{0}$, the flagellar motor force can be estimated to be approximately $0.37 \mathrm{pN}$ in both cases.

\section{Conclusions}

A negative dielectrophoretic force gradient has been used to estimate the force produced by bacterial flagella. The bacteria swim up to a boundary on the electrode at a radius where the magnitude of the dielectrophoretic retarding force equals the motive force. The size of the bacterial collection pattern was compared with a 2-D simulation of the electric field to obtain the force. Using this technique, the motor force of $S$. typhimurium was measured to be approximately $0.37 \mathrm{pN}$. Using negative dielectrophoretic field funnel methods the force generated by the bacteria can be estimated in a quick and non-invasive method. Significantly this can be done in high conductivity media (such as growth media) where the organisms will experience negative DEP over a wide range of frequencies. The method could be used as a relatively simple method for monitoring the real time effects of biological stimuli on the motility of bacteria.

\section{Acknowledgements}

The authors would like to thank Ms M. Robertson and Mr W. Monaghan for electrode fabrication and bacteria preparation, and Dr. N.G. Green for valuable discussions. 


\section{References}

1 Blair DF 1991 The bacterial rotary motor. Nanotechnology 2 123-133.

2 Svoboda K New insights into the workings of the ultimate swimming machine. Biophys. J. 1996, 71, 539-543.

3 Drexler Nanosystems 1992, New York: Wiley.

4 Block SM, Blair DF and Berg HC Compliance of bacterial flagella measured with optical tweezers. Nature 1989, 338, 514-518.

5 Washizu M, Shikida M, Aizawa S-I and Hotani H Orientation and transformation of flagella in electrostatic- field. IEEE Trans. Ind. App. 1992, 28, 1194-1201.

6 Washizu M, Kurahashi Y, Iochi H, Kurosawa O, Aizawa S-I, Kudo S, Magariyama Y and Hotani H Dielectrophoretic measurement of bacterial motor characteristics. IEEE Trans. Ind. App. 1993, 29, 286-294.

7 Berg HC and Turner L Torque generated by the flagellar motor of escherichia coli Biophys. J. 1993, 65, 2201-2216

8 Berry, RM and Berg HC Torque generated by the flagellar motor of escherichia coli while driven backwards Biophys. J. 1998, 76, 580-587

9 Pohl HA Dielectrophoresis 1978, Cambridge: Cambridge University Press.

10 Markx GH, Huang Y, Zhou X-F and Pethig R Dielectrophoretic characterisation and separation of microorganisms. Microbiology 1994, 140, 585-591.

11 Markx GH, Dyda PA and Pethig R Dielectrophoretic separation of bacteria using a conductivity gradient. J. Biotechnol. 1996, 51, 175-180.

12 Jones TB Electromechanics of Particles 1995, Cambridge, MA: Massachusetts Institute of Technology.

13 Huang Y and Pethig R Electrode design for negative dielectrophoresis. Meas. Sci. Tech. 1991, 2, 1142-1146. 
14 Hughes MP and Morgan H Dielectrophoresis of sub-micrometre-scale bioparticles. J. Phys. D: Appl. Phys. 1998, 31, 1-6.

15 Asami K, Hanai T and Koizumi N. Dielectric analysis of Escherichia coli suspensions in the light of the theory of interfacial polarization. Biophys. J. 1980, $31,215-229$.

16 Irimajiri A, Hanai T and Inouye AA dielectric theory of "multi-stratified shell" model with its application to a lymphoma cell. J. Theor. Biol. 1979, 78, 251-269. 


\section{Figures}

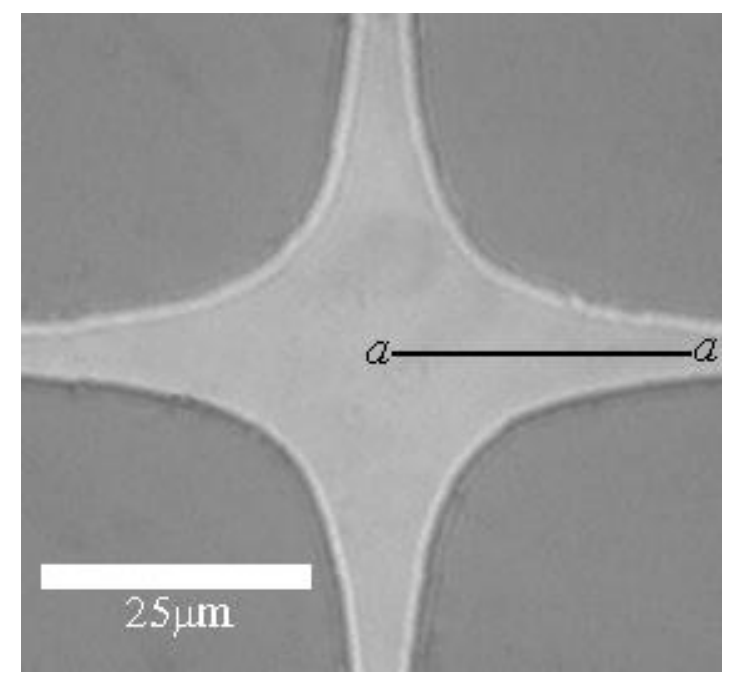

1 Photograph of the electrodes used in the experiment. The electrodes were fabricated on glass slides. The line $a$ - $a$ denotes the radius along which the bacterial collections were measured. Scale bar: $25 \mu \mathrm{m}$. 

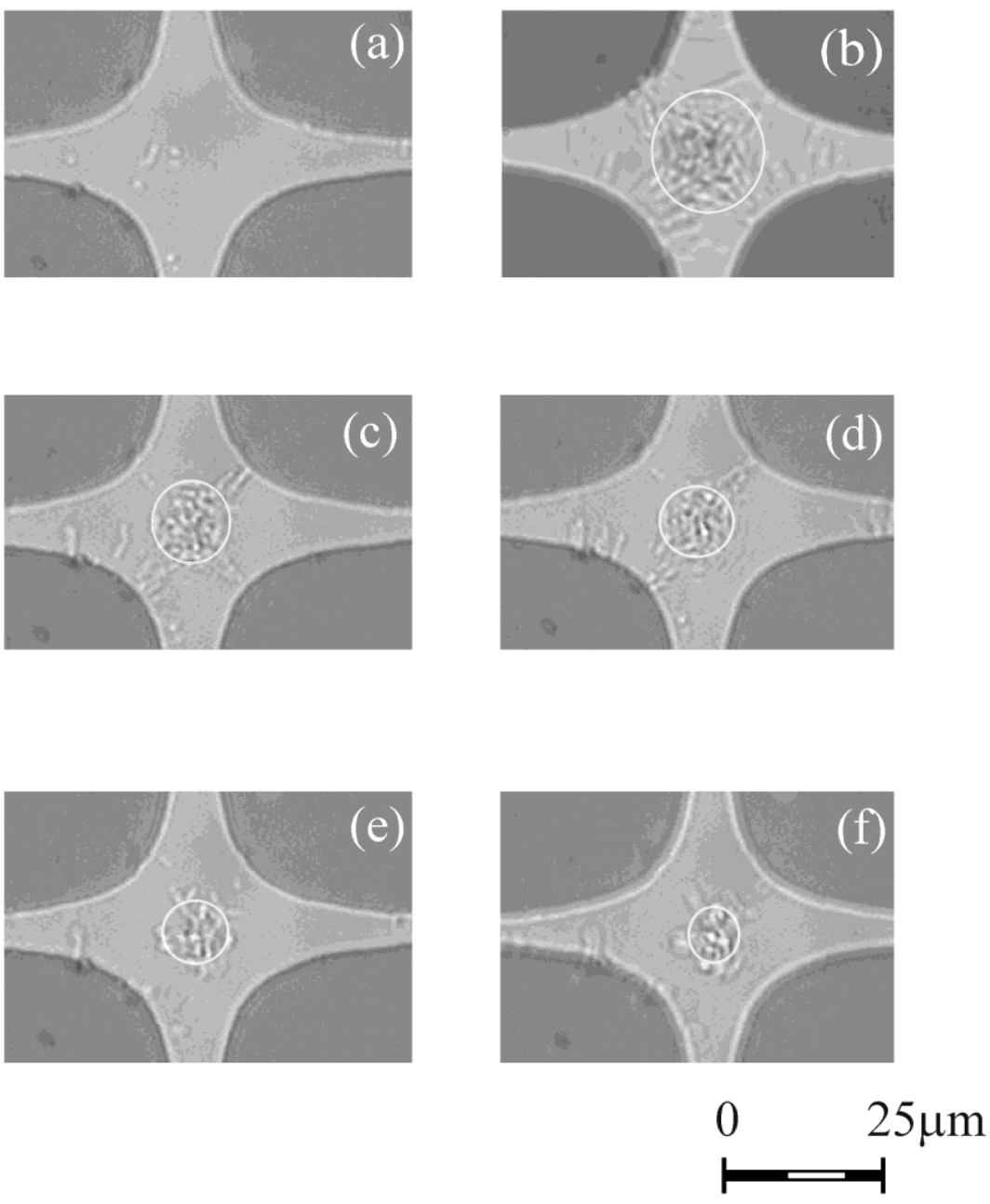

2 Photographs shown how the bacteria collect by negative DEP in the electrode array. Bacteria were suspended in Brain/Heart infusion medium and a $1 \mathrm{MHz}$ signal with potentials in the range 1 to 10 Volts(peak) applied: (a) $1 \mathrm{~V}_{\mathrm{pk}}$, (b) $2 \mathrm{~V}_{\mathrm{pk}}$, (c) $3 \mathrm{~V}_{\mathrm{pk}}$, (d) $4 \mathrm{~V}_{\mathrm{pk}}$, (e) $5 \mathrm{~V}_{\mathrm{pk}}$, (f) $10 \mathrm{~V}_{\mathrm{pk}}$. The white circles indicate the boundary within which bacteria are confined at the level of the electrode. At the higher applied potentials, (e) and (f), a few bacteria are levitated above the electrode surface (they appear outside the drawn circle) and for the purposes of the analysis are discounted. 

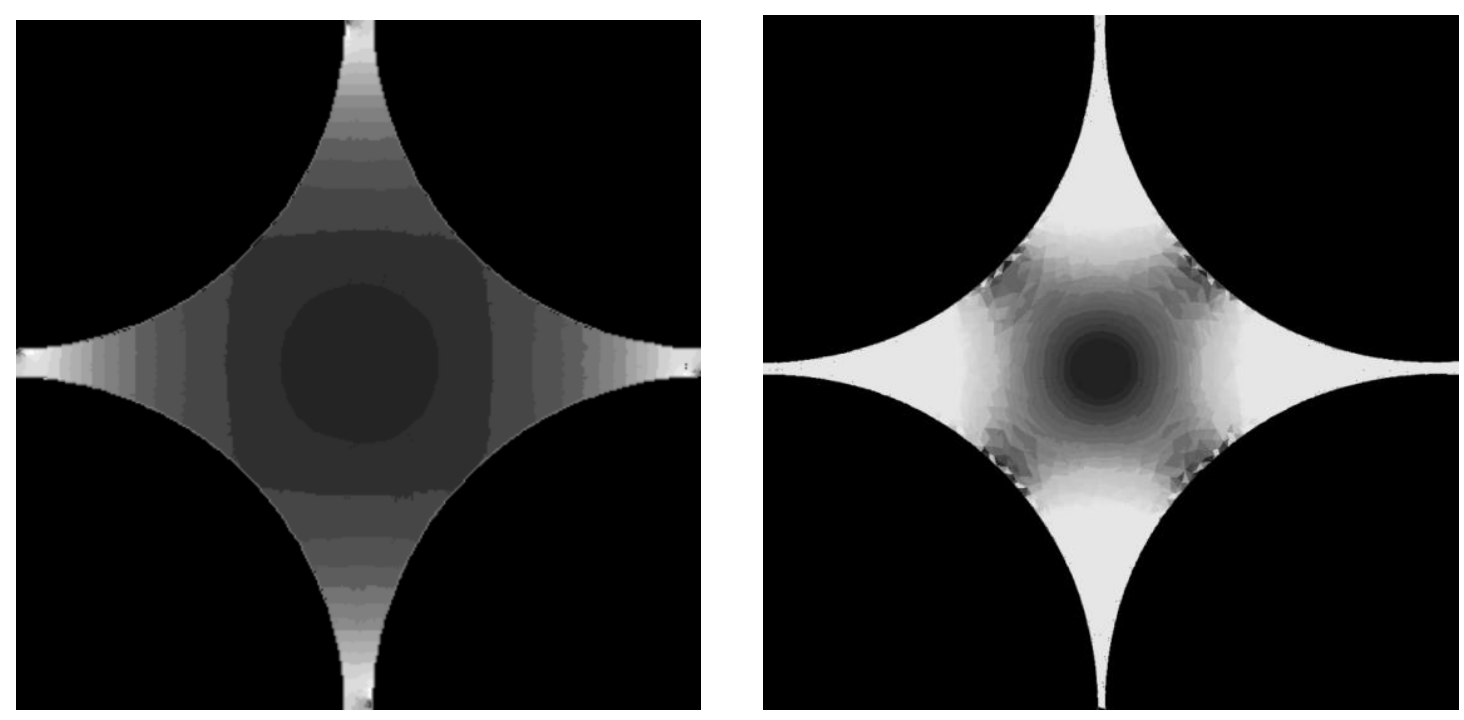

3 (a) A 2-D numerical simulation showing the magnitude of the RMS electric field across the electrode array for an applied potential of $1 \mathrm{~V}_{\mathrm{pk}}$.

(b) A contour plot of the magnitude of $\nabla \mathbf{E}_{r m s}^{2}$ (proportional to the DEP force) between the electrodes with and applied potential of $1 \mathrm{~V}_{\mathrm{pk}-\mathrm{pk}}$ obtained from numerical simulation. There is a similarity between this plot and the bacterial collection patterns shown in figure 2.

\section{Table Legends}

1 Table showing the radius of the experimentally determined bacterial collection patterns together with the calculated values of $\nabla \mathbf{E}_{r m s}^{2}$ obtained from 2-D simulation for the range of applied potentials. 


\begin{tabular}{|c|c|c|c|}
\hline $\begin{array}{c}\text { Applied Potential } \\
\left(\mathrm{V}_{\mathrm{pk}}\right)\end{array}$ & Radius of particle & Calculated & Calculated \\
& collection $(\mu \mathrm{m})$ & $\nabla \mathbf{E}_{r m s}^{2}\left(\mathrm{~V}^{2} \mathrm{~m}^{-3}\right)$ & Force $(\mathrm{pN})$ \\
\hline 1 & no collection & - & - \\
2 & 9 & $1.4 \times 10^{15}$ & 0.33 \\
3 & 5.5 & $1.4 \times 10^{15}$ & 0.33 \\
4 & 4.5 & $1.4 \times 10^{15}$ & 0.33 \\
5 & 3.5 & $2.2 \times 10^{15}$ & 0.5 \\
10 & 2.25 & $5.6 \times 10^{15}$ & 1.28 \\
\hline
\end{tabular}

